\title{
WIRELESS CHARGING SYSTEM WITH A NON-CONVENTIONAL COMPENSATION TOPOLOGY FOR ELECTRIC VEHICLES AND OTHER APPLICATIONS
}

\author{
Ruben Barros Godoy, Emilio Tanowe Maddalena, Glauber de Freitas Lima, Luiz Fernando Ferrari, \\ Vitor Leandro Vieira Torres, João Onofre Pereira Pinto \\ Federal University of Mato Grosso do Sul, Campo Grande - Mato Grosso do Sul, Brazil \\ e-mails: ruben.ufms@gmail.com, emilio.tanowe.maddalena@gmail.com,gdef.lima@gmail.com, luizferrari92@gmail.com, \\ vitorlvt@gmail.com, joao.pinto@ufms.br
}

\begin{abstract}
The proposed paper presents a $500 \mathrm{~W}$, universal input, wireless electric vehicle battery charging system using a non-conventional compensation topology. An algorithm to compensate Loosely Coupled Inductive Power Transfer (LCIPT) systems with such topology is explained. The design and implementation of each power converter, as well as the coils manufacturing process, is briefly covered. Experimental results are shown to validate the expected system behavior.
\end{abstract}

Keywords - Electric Vehicle, Misalignment, Wireless Power Transfer System.

\section{NOMENCLATURE}

$\begin{array}{ll}L_{1} & \text { Primary coil inductance. } \\ R_{1} & \text { Primary coil resistance. } \\ L_{2} & \text { Secondary coil inductance. } \\ R_{2} & \text { Secondary coil resistance. } \\ L_{3} & \text { Primary side inductor compensator. } \\ R_{3} & \text { Primary side inductor compensator resistance. } \\ C_{1} & \text { Primary side series capacitor compensator. } \\ C_{1 P} & \text { Primary side parallel capacitor compensator. } \\ C_{2} & \text { Secondary side series capacitor compensator. } \\ k & \text { Coupling factor. } \\ M & \text { Mutual inductance. } \\ Z_{r} & \text { Reflected impedance. } \\ f r & \text { Reduction factor. } \\ w_{o p} & \text { Maximum power transfer frequency. } \\ w_{d} & \text { Maximum efficiency resonance frequency. } \\ Z_{t} & \text { Total impedance seen by the source. } \\ \mathrm{I}_{1} & \text { Source RMS current. } \\ \mathrm{I}_{2} & \text { Load RMS current. } \\ \mathrm{I}_{\mathrm{p}} & \text { Primary coil RMS current. } \\ \mathrm{I}_{\mathrm{s}} & \text { Secondary coil RMS current. }\end{array}$

\section{INTRODUCTION}

The increasing investments in renewable energies, as well as the efforts to mitigate air pollution, have led recently to a significant interest in electric vehicles (EVs) [1], both from the side of the consumer and the automotive industry. Moreover, the need of autonomy and practicality makes the use of a high-

Manuscript received 20/10/2015. First revision 05/01/2016; second revision $11 / 02 / 2016$. Accepted for publication 11/02/2016, by recommendation of the Regular Section Editor Cassiano Rech. efficiency wireless charging system undoubtedly mandatory for its wide commercial implementation [2]-[4].

A worldwide impact of this technology is expected in a near future, thus a huge concern lies on the power grid system impact that a large number of users would cause to the power quality [5]. That is why high efficiency, high power factor and low harmonic distortion are essential features for such battery chargers.

When designing Loosely Coupled Inductive Power Transfer (LCIPT) systems, high efficiencies can only be achieved by using compensations. Capacitors are usually added in different configurations on both primary and secondary sides to compensate the coils reactive power. There are four classical compensation topologies commonly found in the literature: Series-Series (SS), Series-Parallel (SP), Parallel-Series (PS) and Parallel-Parallel (PP) [6],[7]. Each one of them gives the system a different characteristic in terms of stability and power transfer capability when subjected to misalignment.

In accordance with [8], a non-conventional type of resonance, SPS, is proposed. It is a mix of SS and PS topologies: Series-Parallel compensation on the primary side and Series compensation for the secondary side. It blends the relatively good SS power transfer capability with the PS stability, resulting in a better behavior across a set of parameter variations as well as stability for any range of displacement between coils.

However, this topology does not allow practical implementation, since the presence of the parallel capacitor on the primary stage makes the implementation of the zerovoltage-switching (ZVS) strategy inefficient and dangerous.

The following paper presents a methodology to compensate an LCIPT system with a non-conventional topology - LSPS, which has an inductor in series with the primary SP capacitors - that overcomes the above-mentioned SPS problems. It also presents a complete $500 \mathrm{~W}$, universal input, wireless battery charging system that uses this approach.

The present document is organized in the following way: first, an overview of the implemented prototype features is presented. In the subsequent section, the main equations for the LSPS topology are derived. The actual system implementation process is covered in section V. Next, experimental results are shown. Finally, conclusions and future works are addressed. 


\section{IMPLEMENTED SYSTEM OVERVIEW}

When implementing the following LCIPT system, the main optimization goal was the reduction of the product cost, weight and complexity. By setting the secondary coil voltage to the rated battery voltage in the design phase, the secondary stage was remarkably simplified. The main advantage of this strategy is that it could be easily placed in any EV with little volume and cost for the owner. Along with the LSPS compensation topology, the system intends to transfer rated power efficiently even when subjected to a considerable wide range of misalignment. Figure 1 shows the system organization, while Table I shows its main features.

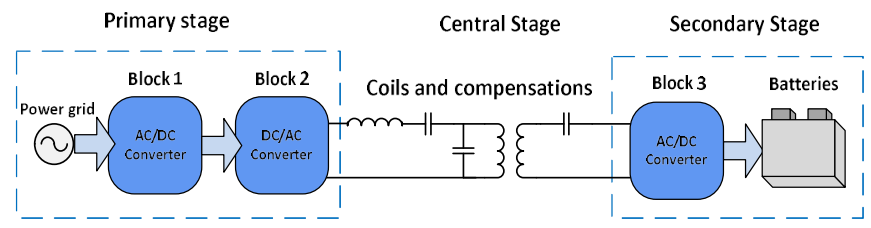

Fig. 1. System architecture.

TABLE I

Implemented System Features

\begin{tabular}{cc}
\hline Item & Value \\
\hline Rated Power & $500 \mathrm{~W}$ \\
\hline Input Voltage & Universal \\
\hline Output Voltage & $36 \mathrm{~V}$ to $60 \mathrm{~V}$ \\
\hline Harmonic Quality & P.F. $\geq 0.98$, THDi $\leq 14 \%$ \\
\hline Weight and Volume & $2.5 \mathrm{Kg}$ and 51 \\
\hline Maximum efficiency & $85 \%$ \\
\hline Manufacturing Cost & $\mathrm{U} \$ 250$ \\
\hline Air Gap & $15 \mathrm{~cm}$ \\
\hline
\end{tabular}

\section{LSPS TOPOLOGY}

In the current section the calculations of the electrical parameters are determined by using Kirchhoff's laws. Figure 2 shows the model used to derive all equations.

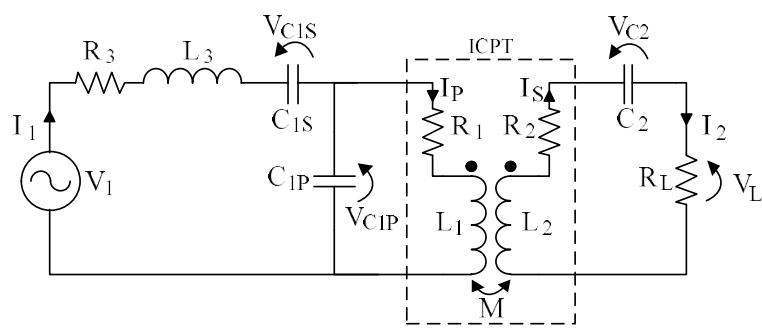

Fig. 2. LSPS compensation model.

To simplify the calculations, first of all, the system is compensated as a PS topology, i.e., assume that $C_{1 S}, L_{3}$ and $R_{3}$ are not considered in the circuit yet. Using Kirchhoff's voltage law for the primary coil ( $L_{1}$ mesh) and for the parallel capacitor ( $C_{1 p}$ mesh), (1) and (2) are obtained:

$$
\begin{gathered}
V_{C_{1 P}}=\left(R_{1}+j \omega L_{1}\right) I_{p}-j \omega M I_{S} \\
I_{c 1 p}=V_{C_{1 P}} j \omega C_{1 p} .
\end{gathered}
$$

Applying Kirchhoff's current law, the primary coil current can be represented by:

$$
I_{p}=I_{1}-I_{C_{1 P}}
$$

Substituting (3) into (2):

$$
I_{p}=I_{1}-V_{C_{1 P}} j \omega C_{1 p}
$$

Now, for the secondary coil, Kirchhoff's voltage law is applied and the secondary current $I_{2}$ is obtained:

$$
\left.I_{2}=\frac{j \omega M I_{p}}{\left[R_{2}+R_{L}+j\left(L_{2} \omega-\frac{1}{C_{2} \omega}\right)\right.}\right] .
$$

Considering $I_{2}=I_{S},(5)$ can be substituted in (1), and $V_{C_{1 P}}$ is simplified as:

$$
V_{C_{1 P}}=\left[\left(R_{1}+j \omega_{1}\right)+\frac{M^{2} \omega^{2}}{\left[R_{2}+R_{L}+j\left(L_{2} \omega-\frac{1}{C_{2} \omega}\right)\right]}\right] I_{p} .
$$

Then, the part between brackets can be separated into reflected impedance $\left(Z_{r}\right)$ and primary coil impedance $\left(Z_{1}\right)$, as observed respectively in (7) and (8). The simplified equivalent circuit is then illustrated in Figure 3.

$$
\begin{gathered}
Z_{r}=\frac{M^{2} \omega^{2}}{\left[R_{2}+R_{L}+j\left(L_{2} \omega-\frac{1}{C_{2} \omega}\right)\right]} \\
Z_{1}=j \omega L_{1}+R_{1}
\end{gathered}
$$

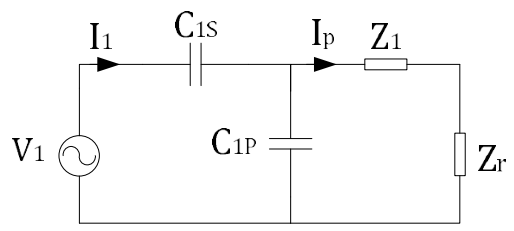

Fig. 3. Simplified circuit for SPS compensation.

The use of both (7) and (8) leads to the total impedance $Z_{t p s}$ seen from $C_{1 S}$, represented as:

$$
Z_{t p s}=\frac{Z_{1}+Z_{r}}{\left(1+\left(Z_{1}+Z_{r}\right) j \mathrm{C}_{1 \mathrm{p}} \omega\right)} .
$$

Looking at the topology as a simple PS compensation, which means that $L_{3}$ and $C_{1 S}$ are still not considered, $C_{1 P}$ must compensate the total reactive part of $Z_{t p s}$, that is:

$$
\operatorname{imag}\left(Z_{t p s}\right)=\frac{L_{1} \omega\left(1-L_{1} C_{1 p} \omega^{2}\right)-\omega C_{1 p}\left(R_{1}+\frac{\omega^{2} M^{2}}{\left(R_{2}+R_{L}\right)}\right)^{2}}{\left(1-L_{1} C_{1 p} \omega^{2}\right)+\omega^{2} C_{1 P}^{2}\left(R_{1}+\frac{\omega^{2} M^{2}}{\left(R_{2}+R_{L}\right)}\right)^{2}}=0
$$

It is important to highlight that the resonance frequency is the same for both primary and secondary stages, and it can be calculated as:

$$
\omega_{d}=\frac{1}{\sqrt{L_{2} C_{2}}}
$$


Finally, from (10) and (11), the resonance capacitance values are obtained according to:

$$
\begin{gathered}
C_{1 p}=\frac{L_{1}}{\left[L_{1}^{2} \omega_{d}^{2}+\left(R_{1}+\frac{\omega_{d}^{2} M^{2}}{\left(R_{2}+R_{L}\right)}\right)^{2}\right]} \\
C_{2}=\frac{1}{\left(L_{2} \omega_{d}^{2}\right)} .
\end{gathered}
$$

As aforementioned, the capacitance calculations were performed considering a PS compensation. Now, a reduction factor is applied intending to reduce the influence of $C_{1 P}$ over the system and, therefore, $C_{1 P}$ is recalculated as $C_{1 P}^{\text {new }}$ :

$$
C_{1 P}^{\text {new }}=f_{r} C_{1 P}
$$

The total PS impedance seen by $C_{1 S}$ is then recalculated as:

$$
Z_{\text {tps }}^{\text {new }}=\frac{Z_{1}+Z_{r}}{1+\left(Z_{1}+Z_{r}\right) j C_{1 P}^{\text {new }} \omega} .
$$

For the entire system total impedance calculation, the inductance $L_{3}$ and $R_{3}$ must also be considered, resulting in the final impedance given by:

$$
Z_{t}=\frac{Z_{1}+Z_{r}}{1+\left(Z_{1}+Z_{r}\right) j C_{1 p} \omega}+j \omega L_{3}+R_{3} .
$$

Consequently, the series capacitor $C_{1 S}$ must be tuned in order to compensate the imaginary part of the total impedance $\left(Z_{t}\right)$.

$$
C_{1 s}=\frac{1}{\omega \cdot \operatorname{imag}\left(Z_{t}\right)}
$$

In order to find the mutual inductance with respect to different displacements between the coils, it was necessary to use Neumann's formula as an approximation [9]. It depends on the number of turns, radius and arrangement of the coils. To simplify, the coupling factor $(k=0.25)$ was empirically obtained for our set of coils from previous experiments. Hence, the mutual inductance can be computed according to:

$$
M=k \sqrt{L_{1} L_{2}}
$$

The choice of $f_{r}$, aiming to reduce displacement effects, is based upon the obtained values presented in the following figures. Figures 4 and 5 show the power delivered to the load $\left(P_{2}\right)$ and the input apparent power $\left(S_{1}\right)$, respectively, against misalignment. These figures provide an effective way to match the transferred power to the total apparent power requested and tolerated by the system when a misalignment occurs.

Notice that Figure 4 shows $P_{2}$ almost constant up to $10 \mathrm{~cm}$ of displacement, and Figure 5 shows that $S_{1}$ is also kept approximately constant even in the absence of a secondary stage when choosing $f_{r}=0.85$. Therefore, a stable behavior with capability of delivering rated power within $10 \mathrm{~cm}$ is expected without the need of closed loop control, or an oversized voltage source when decreasing the primary side parallel capacitor to $85 \%$ of $C_{1 P}$.
Summarizing, low $f_{r}$ values allow high misalignment, with an oversized source, which means that the system behaves more like an SS topology. Whereas, high $f_{r}$ values tend to prohibit rated power transference under the mentioned circumstances, behaving like a PS topology.

Figure 6 briefly shows the behavior of the total impedance when subjected to misalignment for classical compensations (SS, SP, PS and PP) in order to compare them with LSPS. Notice that the total LSPS impedance seen by the source is substantially constant for a wide range of misalignment or even in the absence of the secondary stage.

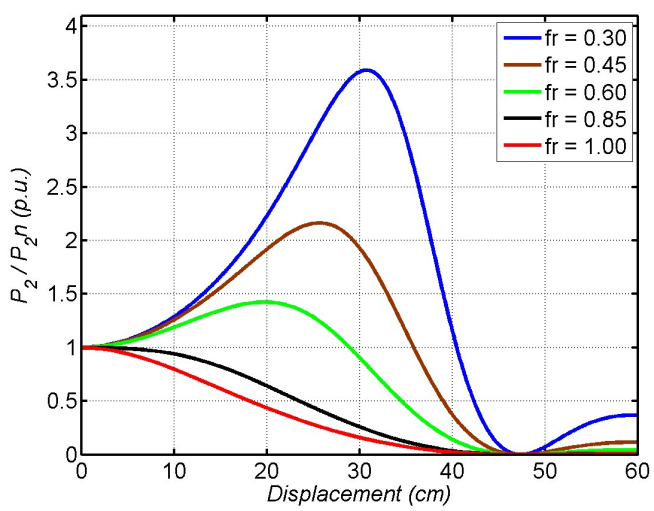

Fig. 4. Output active power versus misalignment.

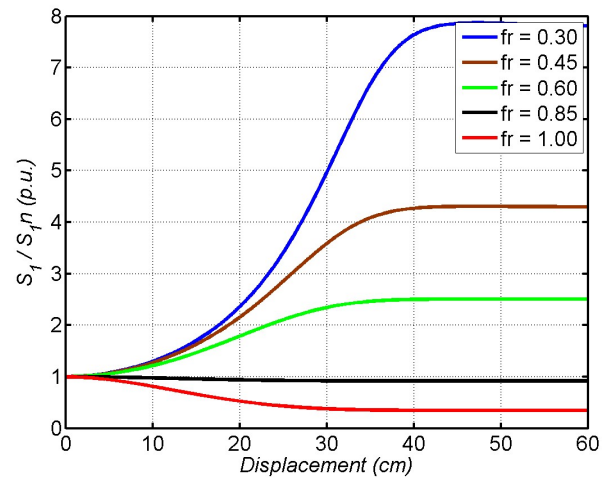

Fig. 5. Input apparent power module versus misalignment.

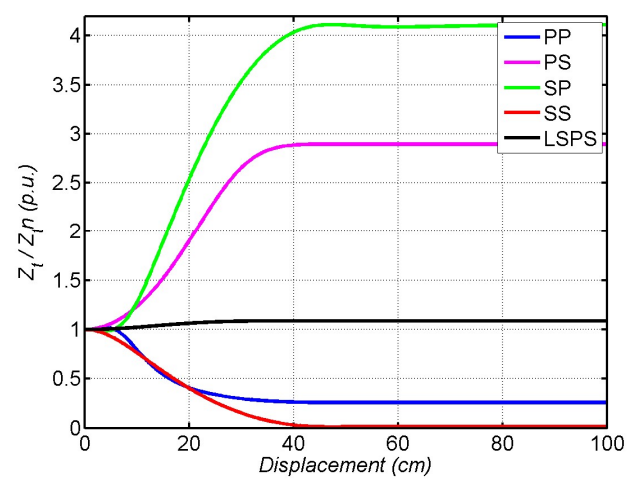

Fig. 6. Total impedance versus misalignment. 
Following, the maximum power transfer frequency and maximum efficiency frequency are derived. Consider that the imaginary part of $Z_{t}$ was zeroed, then, the total impedance seen from the input source $V_{1}$ is totally resistive. Thus, the real part of $Z_{t}$ must satisfy (19) aiming to reach the maximum power transfer.

$$
R_{1}+R_{3}=\frac{\omega_{o p}^{2} M^{2}\left(R_{2}+R_{L}\right)}{\left(R_{2}+R_{L}\right)^{2}+\left(L_{2} \omega_{o p}-\frac{1}{C_{2} \omega_{o p}}\right)^{2}}
$$

Then, the frequency that gives maximum power transfer is simply reduced to:

$$
\omega_{o p}=\frac{\sqrt{\left(R_{1}+R_{3}\right)\left(R_{2}+R_{L}\right)}}{M} .
$$

Nonetheless, $\omega_{o p}$ is not the optimum operating point with respect to the system efficiency. Intending to examine this essential aspect, (21) could be intuitively obtained:

$$
\eta=\frac{R_{L}}{R_{2}+R_{L}+R_{3} \frac{\left|I_{1}^{2}\right|}{\left|I_{2}^{2}\right|}+R_{1} \frac{\left|I_{p}^{2}\right|}{\left|I_{2}^{2}\right|}}
$$

It is possible to find the relation between $I_{I}$ and $I_{P}$ by substituting (6) in (4):

$$
\left|I_{1}\right|=\alpha\left|I_{p}\right|
$$

where:

$$
\alpha=\sqrt{\left(1-\omega_{d}^{2} L_{1} C_{1 p}\right)^{2}+\omega_{d}^{2} C_{1 p}^{2}\left(R_{1}+\frac{M^{2} \omega_{d}^{2}}{R_{2}+R_{L}}\right)^{2}} .
$$

For the information presented in Table II, the calculated $\alpha$ value was 0.357 , meaning that the power loss in $R_{3}$ can essentially be neglected. The ratio of $I_{p}$ to $I_{2}$ is simply obtained from (5) and it is represented by:

$$
\frac{I_{p}}{I_{2}}=\frac{\sqrt{\left(R_{2}+R_{L}\right)^{2}+\left(L_{2} \omega_{d}-\frac{1}{C_{2} \omega_{d}}\right)^{2}}}{\omega_{d} M}=\frac{\left(R_{2}+R_{L}\right)}{\omega_{d} M} .
$$

By substituting (22), (23) and (24) in (21), the system efficiency can be directly represented as:

$$
\eta=\frac{R_{L}}{\left(R_{2}+R_{L}\right)\left(1+\left(R_{3} \alpha^{2}+R_{1}\right) \frac{\left(R_{2}+R_{L}\right)}{\omega_{d}^{2} M^{2}}\right)} .
$$

Finally, it is possible to conclude that the maximum efficiency is obtained when achieving the following condition:

$$
\omega_{d} \gg \frac{\sqrt{\left(R_{3} \alpha^{2}+R_{1}\right)\left(R_{2}+R_{L}\right)}}{M} .
$$

\section{SIMULATIONS}

In order to evaluate the LSPS topology, some preliminary simulations were needed. First of all, primary and secondary self-inductances were determined considering features such as primary input voltage $\left(V_{1}\right)$ presented in Figure 7 , and efficiency, seen in Figure 8. To find the coil design that would result in an adequate and efficient system, primary and secondary self-inductances were 3D-ploted against primary input voltage $\left(V_{1}\right)$ and efficiency. A primary and secondary self-inductances around $280 \mu \mathrm{H}$ and $140 \mu \mathrm{H}$, respectively, associated with a primary voltage around $280 \mathrm{~V}$ (green region) and efficiency around $94 \%$ (dark red region) were then stablished.

After defining $L_{1}, L_{2}$ and $V_{1}$, all parameters were calculated in accordance with the before-mentioned equations and the pre-stablished rated output voltage and output power. The parameters are presented in Table II.

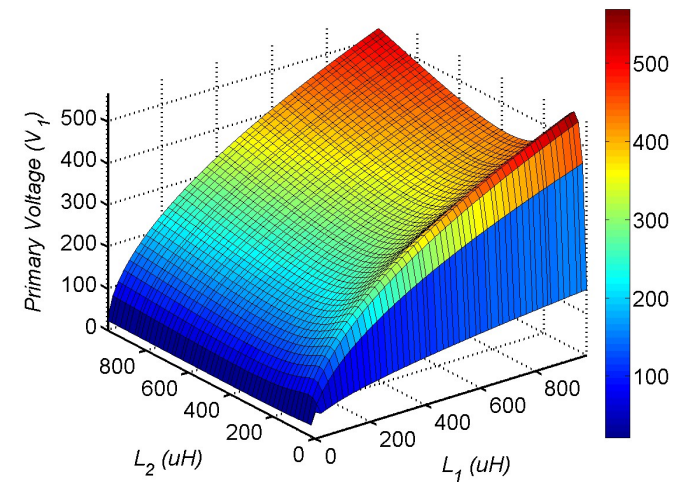

Fig. 7. Self-inductance versus primary voltage.

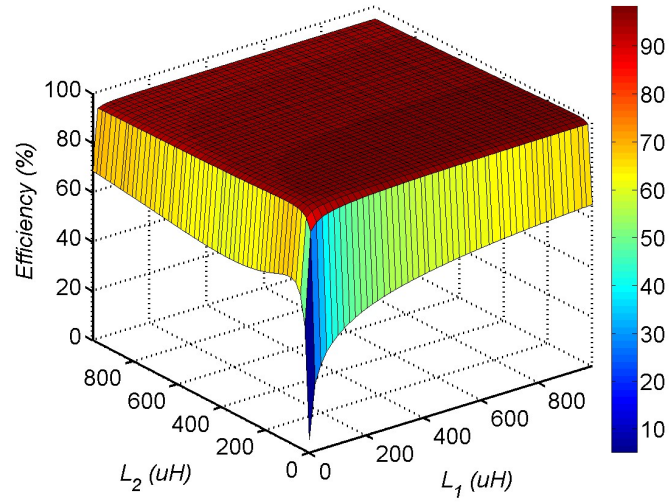

Fig. 8. Self-inductance versus efficiency.

TABLE II

Complete List of Parameters Used for LSPS Topology

\begin{tabular}{cc}
\hline Parameter & Value \\
\hline$R_{L}$ & $4.6 \Omega$ \\
\hline$f$ & $30 \mathrm{kHz}$ \\
\hline$L_{1}$ & $280 \mu \mathrm{H}$ \\
\hline$L_{2}$ & $140 \mu \mathrm{H}$ \\
\hline$L_{3}$ & $160 \mu \mathrm{H}$ \\
\hline$C_{1 S}$ & $57 \mathrm{nF}$ \\
\hline$C_{1 P}$ & $76 \mathrm{nF}$ \\
\hline$C_{2}$ & $201 \mathrm{nF}$ \\
\hline$R_{1}$ & $0.2 \Omega$ \\
\hline$R_{2}$ & $0.2 \Omega$ \\
\hline$R_{3}$ & $0.2 \Omega$ \\
\hline$P_{2}$ & $500 \mathrm{~W}$ \\
\hline$k$ & 0.25 \\
\hline$f_{r}$ & 0.85 \\
\hline$V_{2}$ & $48 \mathrm{~V}$ \\
\hline$V_{1}$ & $280 \mathrm{~V}$ \\
\hline
\end{tabular}


For the choice of $L_{3}$, it was noticed that its value was in direct relation to the voltage across the inductor, the capacitance value $C_{1 P}$ as well as its voltage $V_{C 1 P}$. A value of $L_{3}$ around $160 \mu \mathrm{H}$ was then chosen as it resulted in a feasible value for $C_{1 P}(76 \mathrm{nF})$ and its associated voltage $V_{C 1 P}(177 \mathrm{~V})$.

After defining all the necessary parameters, which can be found in Table II, the system performance was characterized through a set of numerical simulations depicted in Figures 9 and 10 .

As stated in the previous section (Figures 5 and 6), the choice of a proper reduction factor can balance the series and parallel compensations in such a way that the equivalent impedance seen by the source remains practically constant, even when the coils are not aligned. Hence, as expected, even if the power transferred to the load decreases drastically $\left(I_{2}\right)$, the source current $\left(I_{1}\right)$ only decreases approximately $10 \%$. This characteristic (Figure 9) is important because it guarantees the system stability when the secondary coil is not present, which is an intrinsic problem found on the SS topology.

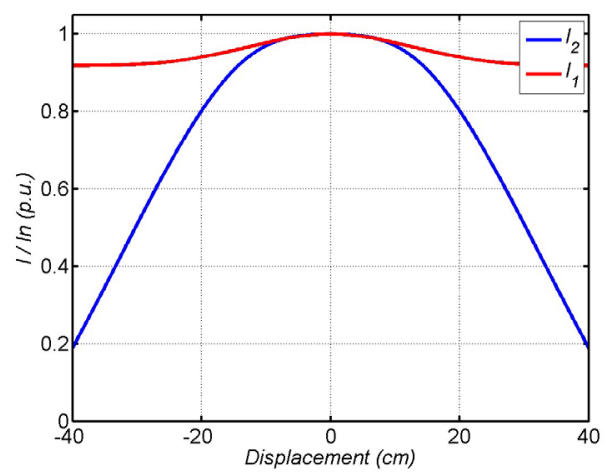

Fig. 9. Source current $\left(I_{1}\right)$ and secondary coil current $\left(I_{2}\right)$ versus coils misalignment.

Moreover, although the power transfer capability is affected by displacement effects, the efficiency of this energy transfer is not. The curve presented in Figure 10 shows that for $10 \mathrm{~cm}$ of misalignment, the requirement for this prototype, the efficiency is essentially constant.

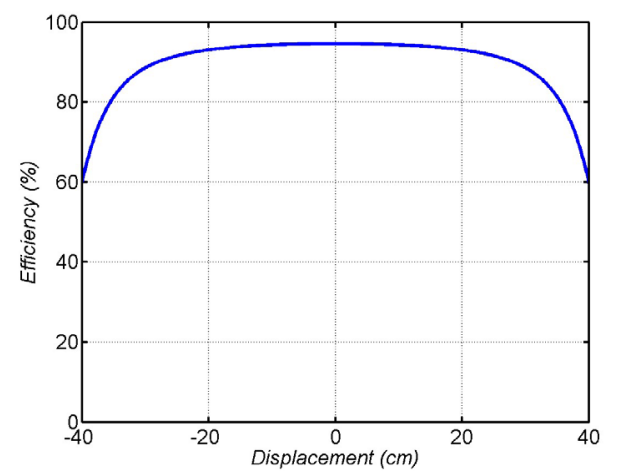

Fig. 10. Central stage efficiency versus coils misalignment.

\section{DESIGNS AND IMPLEMENTATIONS}

\section{A. Primary Stage Power Converters}

As shown in Figure 1, the primary stage is composed of two blocks: an input converter, which rectifies and adjusts the voltage level to the one required by the coils; and an inverter, which adequates the frequency to the resonance design frequency.

Since the primary coil and its compensations require voltage levels around $280 \mathrm{VAC}$ to transmit $500 \mathrm{~W}$, and the grid voltage may vary from 85 to $240 \mathrm{VAC}$, the system is connected to the power grid through a full-wave bridge rectifier and a boost converter, as shown in Figure 11. The control of this block was accomplished by using the UC3854. This integrated circuit has three different loops: a fast inner feedback current loop that corrects the power factor, a slow outer feedback voltage loop that regulates the output voltage, and a feed-forward path that uses the input voltage to compensate disturbances without waiting for it to propagate to the output. The magnitude of this intermediate DC bus voltage controls the power transferred between the primary and secondary coils.

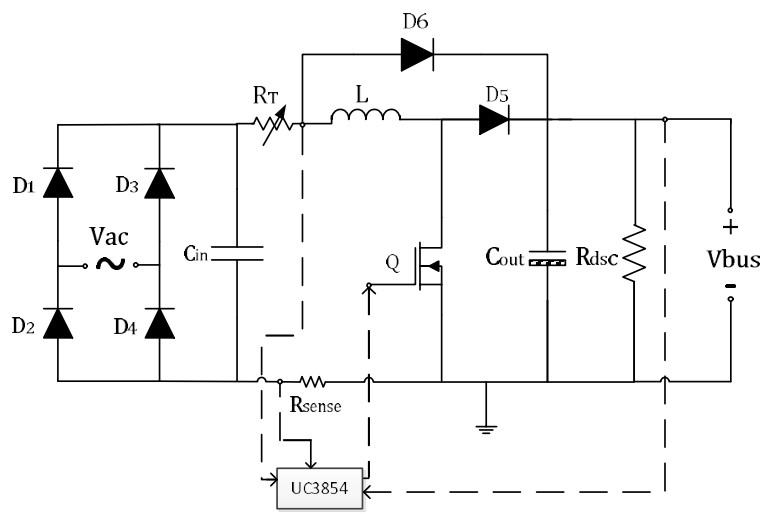

Fig. 11. Input converter schematic.

The DC-AC conversion is performed by an H-bridge inverter composed of four MOSFETs (CMF10120) that switch at $31.5 \mathrm{kHz}$. A dsPIC33F microcontroller (MCU) development board was designed and used to generate the necessary PWM signals. The switching signals pass first through a buffer (MC14503) and then proceed to the gate drivers (HCPL3180). Push buttons were added to enable frequency and duty cycle variations.
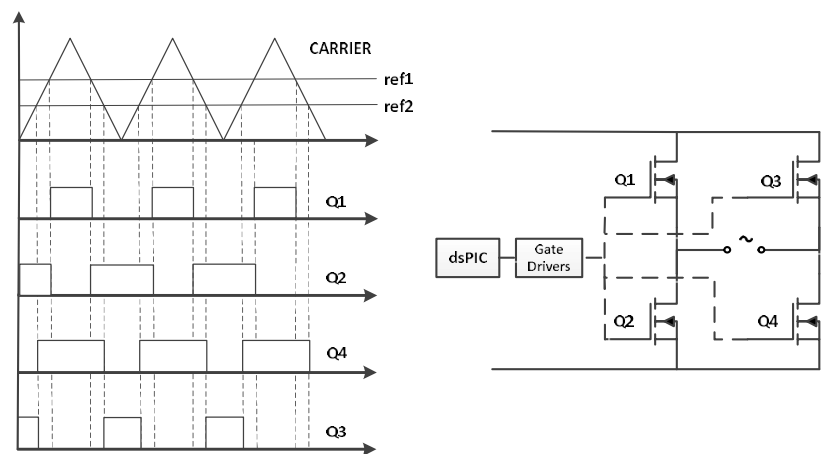

Fig. 12. ZVS switching signals generation. 
Due to its high power dissipation, EMI emission and switching stress, the hard switching technique was avoided [10]-[12] and, in its place, the zero-voltage-switching (ZVS) was introduced. Figure 12 illustrates the process of generating the necessary PWM signals. As stated above, the use of ZVS without the occurrence of short circuits in the parallel capacitor is only possible due to the series inductor compensator. Furthermore, bootstrap capacitors were used to reduce the number of necessary power supplies.

Still on the primary side, a flyback generates the necessary power supplies for the power converters and MCU. It takes as input the boost output voltage and produces three isolated outputs: +19 VDC, +12 VDC and +7 VDC.

\section{B. Coils Design}

In order to achieve high efficiency in the coils stage, it is crucial to obtain low resistances in their design process. Aiming to avoid any skin effect, the AWG choice of the litz wire was based according to (27). For a $31.5 \mathrm{kHz}$ operating frequency, AWG21 was used.

where:

$$
\delta=\sqrt{\frac{\rho}{\pi f \mu_{0}}}
$$

$\delta$ - skin depth;

$\rho$ - copper resistivity;

$f$ - operating frequency;

$\mu_{0}$ - vacuum magnetic permeability.

As the air has a constant magnetic permeability, the inductance is a function of the chosen geometry. Instead of other configurations, a planar shape was chosen considering better coupling factor across misalignments, while the circular disposition was selected due to its symmetry when subjected to misalignments. Using (28) to (30), the inductance for this geometry can be calculated:

where:

$$
\begin{gathered}
L=\mu_{0} N^{2} R_{\text {avg }}\left(\ln \frac{16 R_{\text {avg }}}{d}-2\right) \\
d=2 \sqrt{\frac{N S_{\text {litz }}}{\pi}} \\
S_{\text {litz }}=K_{s} N_{\text {litz }} \pi R_{\text {awg }}^{2}
\end{gathered}
$$

$N$ - number of turns;

$R_{\text {avg }}$ - average radius;

$d$ - equivalent diameter;

$S_{\text {litz }}$ - litz wire real cross-section;

$R_{a w g}$ - choosen AWG radius;

$N_{\text {litz }}$ - number of litz wires;

$\mathrm{K}_{\mathrm{S}}$ - adjusting factor to account for imperfections in the manufacturing process.

To obtain a feasible design for the coils, it is needed to match physical constraints to theoretical requested inductances. In this application, the coil maximum diameter was limited to $50 \mathrm{~cm}$. Figure 14 presents a picture of the system's primary coil, which is composed of 14 wires of AWG 21. A flowchart that describes the design algorithm is shown in Figure 13:

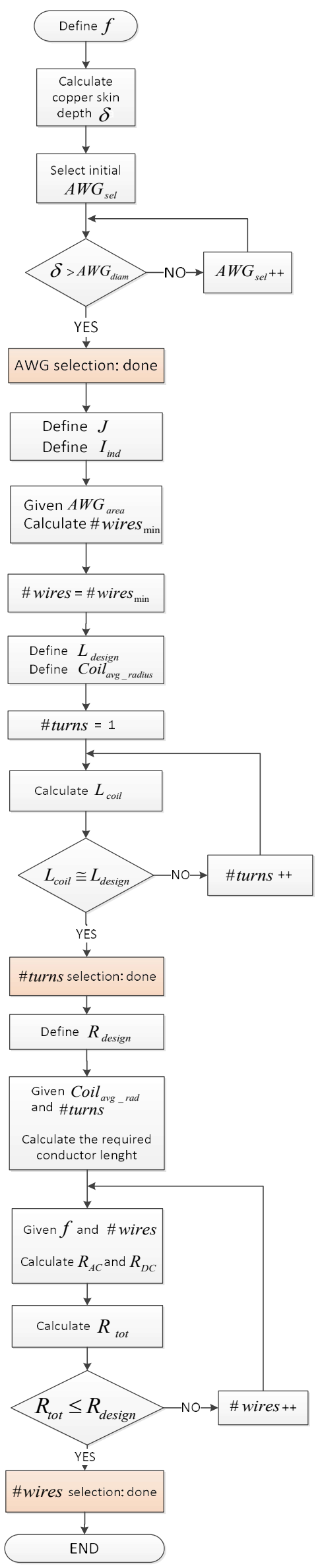

Fig. 13. Coils design flowchart. 
where:

$f$-operating frequency;

$\delta$ - skin depth;

$A W G_{\text {sel }}-\mathrm{AWG}$ selected value;

$A W G_{\text {diam }}$ - AWG diameter;

$A W G_{\text {area }}-\mathrm{AWG}$ cross-sectional area;

$J$ - maximum current density;

$I_{\text {ind }}$ - coil's operating current;

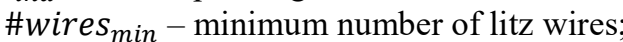

\#wires - final number of litz wires;

$L_{\text {design }}$ - desired inductance value;

$L_{\text {coil }}$ - expected inductance value;

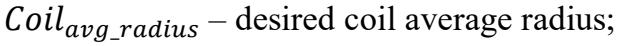

\#turns - final coil's number of turns;

$R_{\text {design }}-$ desired coil's resistance value;

$R_{D C}-$ coil's DC resistance;

$R_{A C}$ - coil's AC resistance;

$R_{\text {tot }}$ - coil's total resistance.

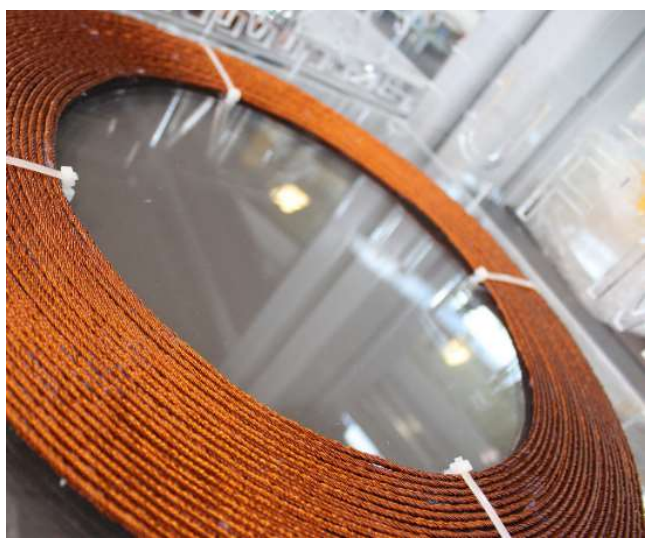

Fig. 14. Primary coil picture: 14 wires, AWG 21 .

\section{Secondary Stage Power Converter}

On the secondary side, a synchronous rectifier with four $\mathrm{N}$-channel MOSFETs (IRFP4468) was used to charge the battery bank. To avoid using another microcontroller or complex driving circuits, the switches are triggered by using high frequency transformers. The criterion to select the turn ratio is given by:

where:

$$
\text { Turns ratio }=\frac{N_{p}}{N_{S}}=\frac{V_{\text {in } \_p k}}{V_{G S \_} \max }
$$

$N_{p}$ - primary number of turns;

$N_{s}-$ secondary number of turns;

$V_{\text {in_pk }}-$ peak value of the input voltage;

$V_{G S_{-} \max }-$ MOSFET maximun $V_{G S}$.

Since the selected switch has $V_{G S \_\max }$ of $20 \mathrm{~V}$ and the voltage peaks were near $75 \mathrm{~V}$, two $4: 1$ transformers were manufactured. Figure 15 presents the complete schematic.

The fact that the battery imposes its voltage on the rectifier may cause a short circuit with the coils alternating voltage. Therefore, a diode is needed to block any reverse current coming from the battery. Aiming to increase efficiency, this diode was replaced by a fifth MOSFET (Q5), which is triggered by a dedicated IC (IR1167).

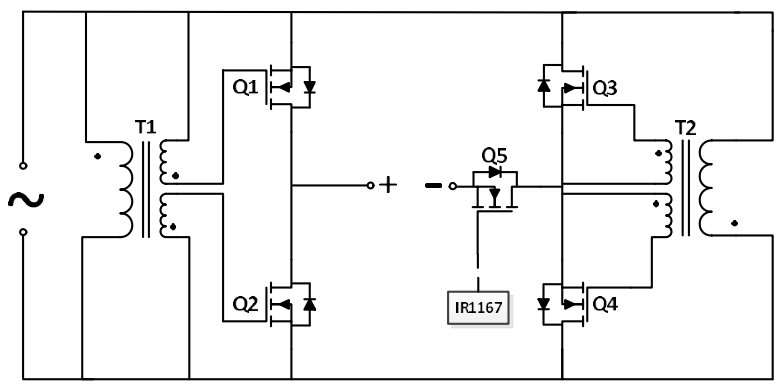

Fig. 15. Secondary side synchronous rectifier.

Apart from being simple, this topology also presented efficiency up to $98 \%$ at $500 \mathrm{~W}$, which was very satisfactory for this application.

\section{EXPERIMENTAL RESULTS}

A picture of the complete system is presented in Figure 16, which is organized as follows: 1 - primary stage converters and compensations with total volume of 3.5 1,2 - pair of coils with approximately $25 \mathrm{~cm}$ of radius; 3 - secondary side converter with volume of 1.51 , and 4 - lead-acid batteries. It is important to mention that, since a flyback converter is incorporated in block 1, all the efficiency measurements present in this section already take into account auxiliary circuits and power supplies.

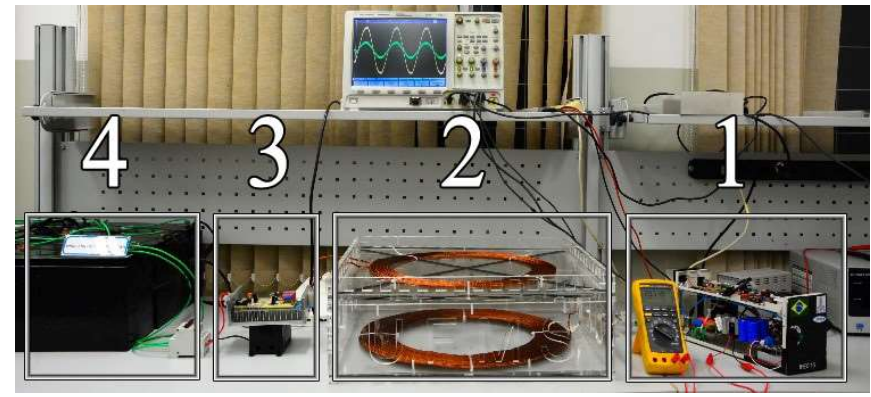

Fig. 16. Complete system picture.

When delivering rated power, the prototype power factor is always greater than 0.98 for the entire range of input voltages ( $85 \mathrm{VAC}$ to $240 \mathrm{VAC}$ ). Figure 17 presents the input voltage and current waveforms when transferring $480 \mathrm{~W}$. In such case, the current THD was calculated considering up to the 30th harmonic order, resulting in a value of $13 \%$.

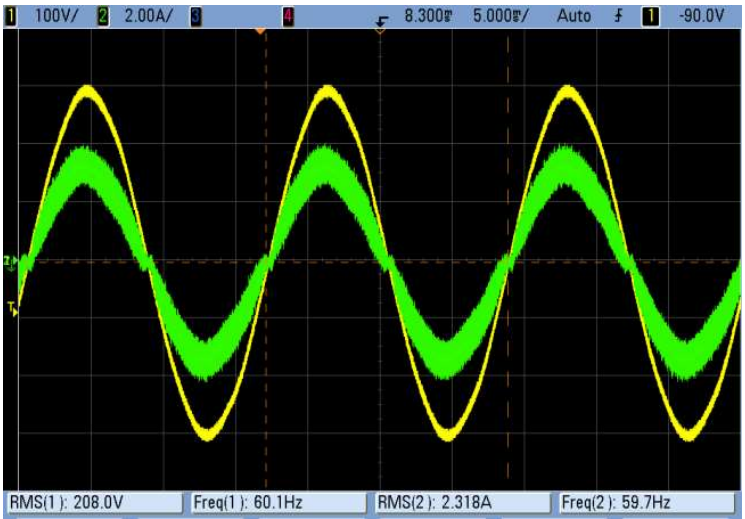

Fig. 17. Input voltage (yellow) and input current (green) waveforms. 
In Figure 18, each color is associated with a different label as explained in the following. The batteries constant voltage (V_BAT) is shown in yellow. In green (I_BAT), the batteries charging current is noticed. This current is rectified and consequently oscillates at twice the inverter frequency (31.5 $\mathrm{kHz}$ ). The inverter output voltage (V_INV) is presented in blue. It is clearly observable the presence of three levels as a consequence of zero voltage switching. Finally, the inverter output current is presented in red (IIINV). Two transient periods are present at each semi cycle of this current. The shortest one happens during the freewheeling period when the converter sets zero voltage over the load. During this period the current decreases around zero. It represents a resonance that conducts to a turn-on zero current switching. The second period (largest one) is associated with the power transfer itself.

Figure 19 shows two of the before mentioned results (synchronous rectifier output) together with voltage and currents across the synchronous rectifier input. Notice that the secondary coil (I_OUT in red) delivers a $31.5 \mathrm{kHz}$ sinusoidal current, whereas the voltage has a quasi-squared shape imposed by the batteries bank.

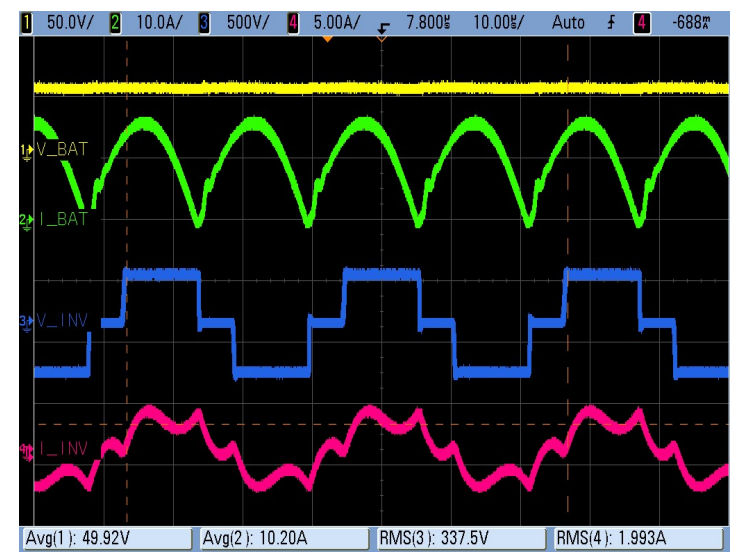

Fig. 18. Inverter and synchronous rectifier output waveforms. V_BAT (yellow) battery voltage; I_BAT (green) battery current; $\mathrm{V}^{-}$INV (blue) inverter output voltage; I_INV (red) inverter output current. Input voltage of $120 \mathrm{VAC}$ and output power of $500 \mathrm{~W}$.

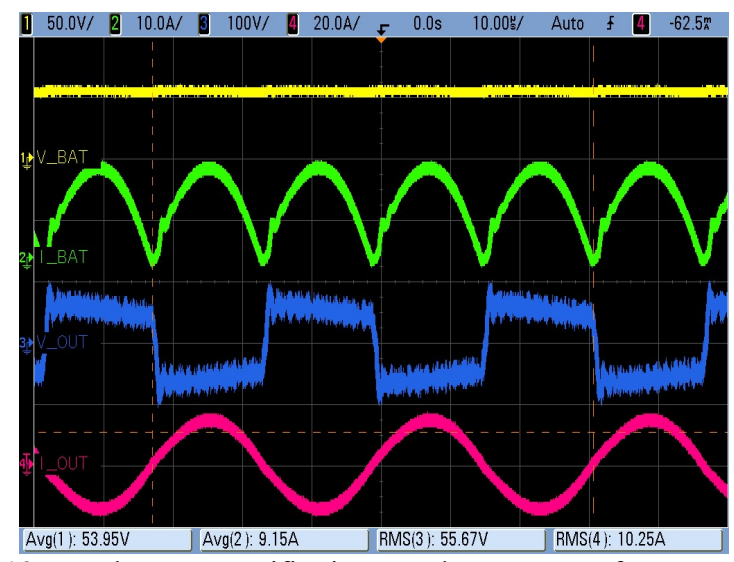

Fig. 19. Synchronous rectifier input and output waveforms. V BAT (yellow) battery voltage; I_BAT (green) battery current; V_OUT (blue) secondary compensated coil output voltage; I_OUT (red) secondary compensated coil output current. Input voltage of 120 VAC and output power of $500 \mathrm{~W}$.
Several tests were conducted in order to analyze the overall efficiency. Some of these results were selected and they are presented in the following. In advance, it is important to mention that a discussion about total and distributed losses is made at the end of this section.

In Figure 20 the system is operating at rated power and the input voltage is varying. The continuous lines represent measured values whereas the dotted lines represent extrapolated values based on the curves tendency. It is worth mentioning that these essays were performed for two different frequencies $(30.5 \mathrm{kHz}$ and $31.5 \mathrm{kHz})$. The lower one $(30.5$ $\mathrm{kHz})$ is the design frequency, theoretically applied for all calculations. The second one $(31.5 \mathrm{kHz})$ is the effective resonance frequency, experimentally found out after the implementation of the compensators. For the results presented in Figure 20, there was no displacement between the coils and the air gap was of 15 centimeters. Essentially, the collected results proved that the higher frequency presented a small gain of efficiency as expected.

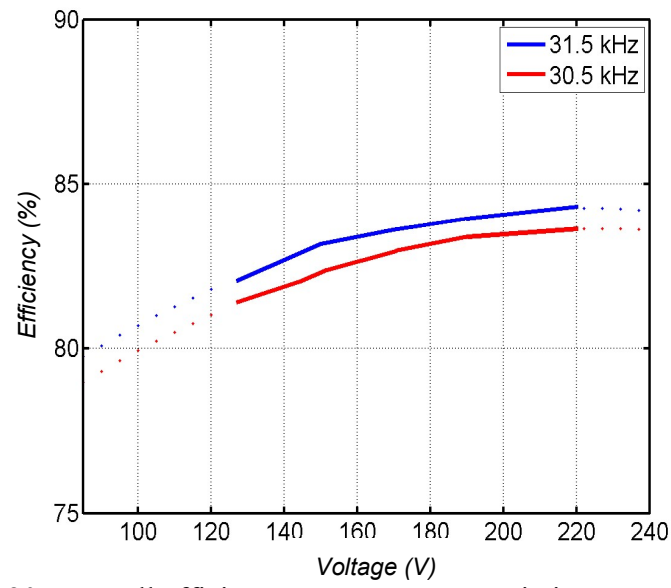

Fig. 20. Overall efficiency across voltage variations.

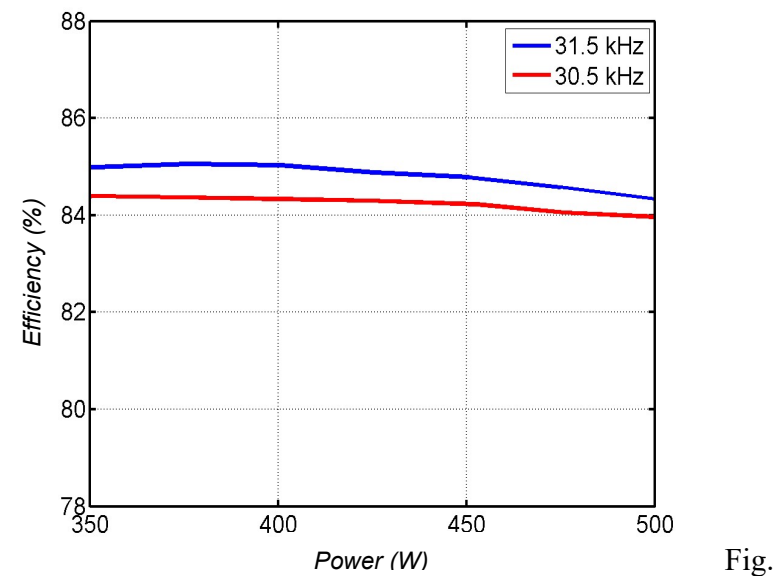

Fig. 21. Overall efficiency versus output power - input voltage of $220 \mathrm{~V}$.

In order to evaluate the power loss profile, efficiency was measured by output power variations. Figure 21 presents the overall efficiency considering an output power range from 350 $\mathrm{W}$ to $500 \mathrm{~W}$, input voltage of $220 \mathrm{VAC}$ and no misalignment between coils. In both situations, the battery voltage was around its rated value of $48 \mathrm{~V}$. The tendency to reduce 
efficiency if the output power increases suggests that conduction losses are prevalent throughout the circuit.

Finally, Figure 22 presents the efficiency for a misalignment of $10 \mathrm{~cm}$ and input voltages of 220 VAC. The decrease of efficiency due to displacement was somewhat modest if compared to other topologies. This result was important to prove the stability of the system throughout coils displacement in accordance with the theoretical approach presented in previous sections.

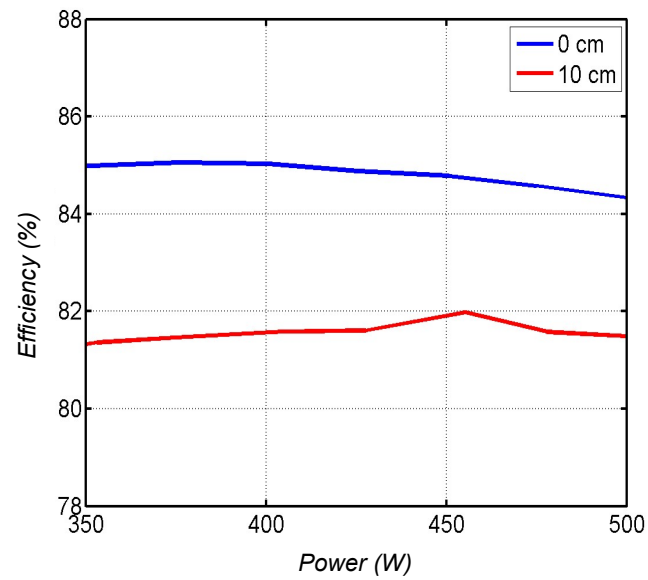

Fig. 22. Overall efficiency versus output power for misalignment of $10 \mathrm{~cm}$ - input voltage $220 \mathrm{~V}$.

The individual efficiency of each power stage was also measured. The system analysis was performed under the following conditions: input voltage of $220 \mathrm{VAC}$, rated output power $(500 \mathrm{~W})$ and aligned coils with $15 \mathrm{~cm}$ of air gap between them. The power losses distribution along each block can be seen in Figure 23.

Evidently, these results confirm that the first stage efficiency must be improved in order to guarantee less energy dissipation. Some attractive topologies for high efficiency boost rectifiers are thoroughly described in the literature [13]. Still regarding to decrease losses, an alternative could be both redesigning the coils with lower parasitic resistances [14] and increasing the coupling factor by either more appropriate coils geometry or using ferrite on them [15].

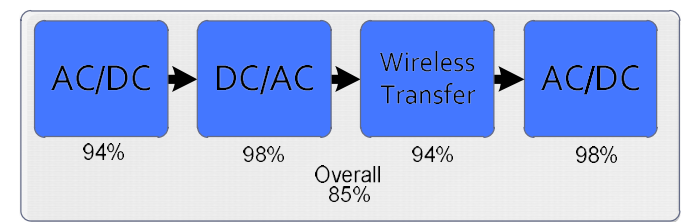

Fig. 23. Efficiency analysis on each subsystem.

\section{CONCLUSION}

A complete $500 \mathrm{~W}$ wireless battery charging system was fully designed and implemented. Its main characteristics are: maximum wall-to-battery efficiency of $85 \%$, universal input, power factor greater than 0.98 , THD lower than $14 \% ; 15 \mathrm{~cm}$ of air gap between coils, and capable of transferring rated power even when subjected to $10 \mathrm{~cm}$ of misalignment in any direction. Moreover, since expensive ferrite bars were not used to increase the coupling factor, the total manufacturing cost for 5000 units was kept low, around U\$250. The present prototype applications can also be extended to systems besides EVs.

The secondary stage was remarkably simplified in terms of circuitry and optimized in terms of volume, considering that it is only composed of the secondary coil, a series capacitor compensator, and a synchronous rectifier with a passive MOSFETs triggering circuit.

An extensive study of the resonance compensation phenomenon is mandatory to reach high efficiencies in the wireless power transfer stage. Furthermore, the choice of the compensation topology is what defines the system behavior when facing misalignments and its operation outside the design frequency. The LSPS compensation, with the careful selection of the reduction factor, was the key to enable rated power transfer even when the coils were not perfectly matched, and to enable the use of the ZVS switching strategy in the $\mathrm{H}$-bridge inverter.

It is important to maintain a high degree of precision in the resonance design process, i.e., when designing the coils and selecting the compensator values. Nevertheless, since some resonance bands may be narrow, the final complete system's resonance frequency should be found empirically to ensure maximum efficiency.

Lastly, future works include the design of a bridgeless boost converter for the input stage and the feedback of the secondary voltage and current information to the primary side MCU. This could lead the complete charging process to be autonomous and, thus more reliable.

\section{ACKNOWLEDGMENTS}

The authors thank everyone that contributed to the present work and also recognize the following people as authors of this paper: Alison França Queiroz da Costa, André Acosta Amaral, Caio Guilherme da Silva Moraes, Filipe de Castro e Silva, Gabriel de Figueiredo Gentil, Hugo Gonçalves Bertolassi, Janaina Luiz Ramires, José de Arimatéia Olimpio Filho, Kássio José Lara de Rezende, Larissa Rodrigues Souza, Márcio Luiz Magri Kimpara, Renata Rezende da Costa Reis Kimpara, Rodrigo Alves Ribeiro Calunga and Tiago Henrique de Abreu Mateus.

\section{REFERENCES}

[1] M. do C. B. P. Rodrigues, J. G. de Oliveira, A. A. Ferreira, P. G. Barbosa, H. A. C. Braga, "Grid connection of Electric Vehicles for Battery Charging: a Survey", Eletrônica de Potência - SOBRAEP, vol. 19, no. 2, pp. 193-207, May 2014.

[2] C. C. Chan, "The State of the Art of Electric, Hybrid, and Fuel Cell Vehicles", Proc. of the IEEE, vol. 95, no. 4, pp. 704-718, Apr. 2007.

[3] T. Imura, H. Okabe, Y. Hori, "Basic experimental study on helical antennas of wireless power transfer for Electric Vehicles by using magnetic resonant couplings", in Proc. of the Vehicle Power and Propulsion Conference, pp. 936-940, 2009.

[4] R. C. Fernandes, A. A. de Oliveira Jr., "Selected Topics on the State-of-the-Art of Inductive Power Transfer", 
Eletrônica de Potência - SOBRAEP, vol. 19, no. 1, pp. 58-71, Feb. 2014.

[5] S. A. Sabki, N. M. L. Tan, "Wireless Power Transfer for Electric Vehicle", in IEEE 8th International Power Engineering and Optimization Conference, pp. 41-46, 2014.

[6] C. Wang, O. H. Stielau, G. A. Covic, "Design Considerations for a Contactless Electric Vehicle Battery Charger", IEEE Transaction on Industrial Electronics, vol. 52, no. 5, pp. 1308-1314, Oct. 2005.

[7] S. Jeong, J. Jung, Kim, K.A.; J. Kim, “Analytical investigation of optimal wireless power transfer topology for electric vehicles", in IEEE PELS Workshop on Emerging Technologies: Wireless Power (WoW), pp. $1-5,2015$.

[8] J. L. Villa, J. Sallan, A. Lombart, J. F. Sanz, "HighMisalignment Tolerant Compensation Topology for ICPT Systems", IEEE Transactions on Industrial Electronics, vol. 59, no. 2, pp. 945-951, Feb. 2012.

[9] J. L. Villa, J. Sallan, A. Llombart, J. F. Sanz, "Optimal Design of ICPT Systems Applied to Electric Vehicle Battery Charge", IEEE Transactions on Industrial Electronics, vol. 56, no. 6, pp. 2140-2149, June 2009.

[10] M. A. Kazemi, E. Adib, H. Farzanehfard, "A New forward type zero voltage switching inverter", Power Electronics and Drive Systems Technology (PEDSTC), pp. 477-482, 2012.

[11] M. Mezaroba, R. L. Klein, R. J. M. dos Santos, A. F. de Paiva, A. L. Batschauer, "Step-up/Step-down ZVZCS PWM DC-DC Converter with Active Clamping", Eletrônica de Potência - SOBRAEP, vol. 17, no. 2, pp. 503-512, May 2012.

[12] A. L. Kirsten, F. G. Carloto, T. H. de Oliveira, J. G. Roncalio, M. A. D. Costa, "Phase-Shift Design Methodology for the Dab Converter", Eletrônica de Potência-SOBRAEP, vol. 19, no. 3, pp. 231-240, Aug. 2014.

[13] L. Huber, J. Yungtaek, M. M. Jovanovic, "Performance Evaluation of Bridgeless PFC Boost Rectifiers", IEEE Transactions on Power Electronics, vol.23, no. 3, pp. 1381-1390, May 2008.

[14] H. G. Parke, U. T. Parke, "Minimum-resistance coil design", Electrical Engineering, vol. 80, no. 11, pp. 846848, Nov. 1961.

[15] M. Budhia, G. A. Covic, J. T. Boys, "Design and Optimization of Circular Magnetic Structures for Lumped Inductive Power Transfer Systems", IEEE Transactions on Power Electronics, vol. 26, no. 11, pp. 3096-3108, Nov. 2011.

\section{BIOGRAPHIES}

Ruben Barros Godoy, was born in Campo Grande, MS, Brazil. He received the B.S. degree from Federal University of Mato Grosso do Sul, Brazil, in 2004. The M.S. degree was obtained from the same University in 2006. Ph.D. degree from São Paulo State University, at Ilha Solteira, Brazil. He currently holds a faculty position in Federal University of Mato Grosso do Sul at Campo Grande, MS, Brazil. His main publications are related in power electronics and artificial intelligence. Currently conducts research on parallel inverters, smart micro-grids, optimal power management, and wireless power transfer.

Emilio Tanowe Maddalena, was born in Campo Grande, Brazil, in 1993. He is currently an electrical engineering student at the Federal University of Mato Grosso do Sul. His interests include modeling of dynamic systems, control theory and embedded systems.

Glauber de Freitas Lima, was born in Goiânia, Brazil, in 1992. He is currently an electrical engineering student at the Federal University of Mato Grosso do Sul. His interests include electromagnetism, power electronics, control theory and renewable energy.

Luiz Fernando Ferrari, was born in Fátima do Sul, Brazil, in 1992. He is currently an electrical engineering student at the Federal University of Mato Grosso do Sul. His interests include power electronics, control theory and embedded systems.

Vitor Leandro Vieira Torres, was born in Paranaíba, Brazil, in 1993. He is currently an electrical engineering student at the Federal University of Mato Grosso do Sul. His interests include embedded systems, hardware/software integration, computer architecture and control theory.

João Onofre Pereira Pinto, was born in Valparaiso, SP, Brazil. He received the B.S degree from São Paulo State University at Ilha Solteira, SP, Brazil, in 1990, the M.S. degree from Federal University of Uberlândia, MG, Brazil, in 1993, and the Ph.D. degree from the University of Tennessee, Knoxville, in 2001. He currently holds a faculty position at Federal University of Mato Grosso do Sul at Campo Grande, MS, Brazil. His research interests include signal processing, neural networks, fuzzy logic, genetic algorithm, and wavelet applications to power electronics, PWM techniques, drives, and electric machines control. 